\title{
Fault Diagnosis of Power Transformer Based on Memristive Neural Network
}

\author{
Xun Wang \\ College of Automation, Tianjin University of Technology, Tianjin 300384, China
}

Keywords: Transformer, Memristor, Fault diagnosis, Neural network

\begin{abstract}
Memristive neural network is applied to fault diagnosis of power transformer in this paper. A model of memristive neural network built by MATLAB Simulink can be used to diagnose and analyze the failure information. Experimental results show that the proposed method can achieve better results and has a certain value.
\end{abstract}

\section{INTRODUCTION}

Oil-immersed power transformer is an important equipment of modern power systems. A variety of reasons including over-voltage, lightning, insulation aging, discharge, man mistakes can cause transformer faults. Seriously, it can make a severe damage to power systems. Diagnosing faults of transformer timely and accurately, which has important significance for operation of power systems.

This paper takes the power transformer as the research object. In order to quickly identify and diagnose faults, a memristive neural network is applied to fault diagnosis of transformer. In treating the problem of pattern recognition, memristive neural network can compute in parallel and the operating speed is quick. It can greatly improve the efficiency of fault diagnosis. After example validating, this method can effectively diagnose faults of power transformer.

\section{FAULT DIAGNOSIS METHOD OF MEMRISTIVE NEURAL NETWORK}

\subsection{Model of memristive neural network}

In 1971, Cai Shaotang put the resistance of memory referred to as memristor, and defined its mathematic model. The memristor is known as the fourth basic passive component. In 2008, after Cai Shaotang proposed the memristor 30 years, HP finally developed the actual physical model of TiO2-based memristor. Memristor is a passive component change the resistance value through the inflow of voltage or current. The characteristic is that it can "memory" the last value after power-off.

In this study, memristor is used as the weight of neural network, by controlling the voltage at both ends of memristor to modify memristor's resistance, which is adjusting weights.

The overall design of memristive neural network is shown in Figure 1. This neural network consists of input layer and output layer, which simulates the self organization feature function of human brain neural network system. The input layer is a one-dimensional matrix, neuron number is 3. The output layer is a two-dimensional node matrix, which consists of neurons arranged in a square planar $6 \times 6$ Neurons in the input layer and the output layer connect together by memristors. The input an output layer is shown in Figure 2. The function of winning neuron judgment module is to find the closest point (the winning neuron) between the input vector and the weight, and train weights. The winning neuron judgment module is shown in Figure 3. Finally, the training result will display to the user in the form of images. The display module is shown in Figure 4. 


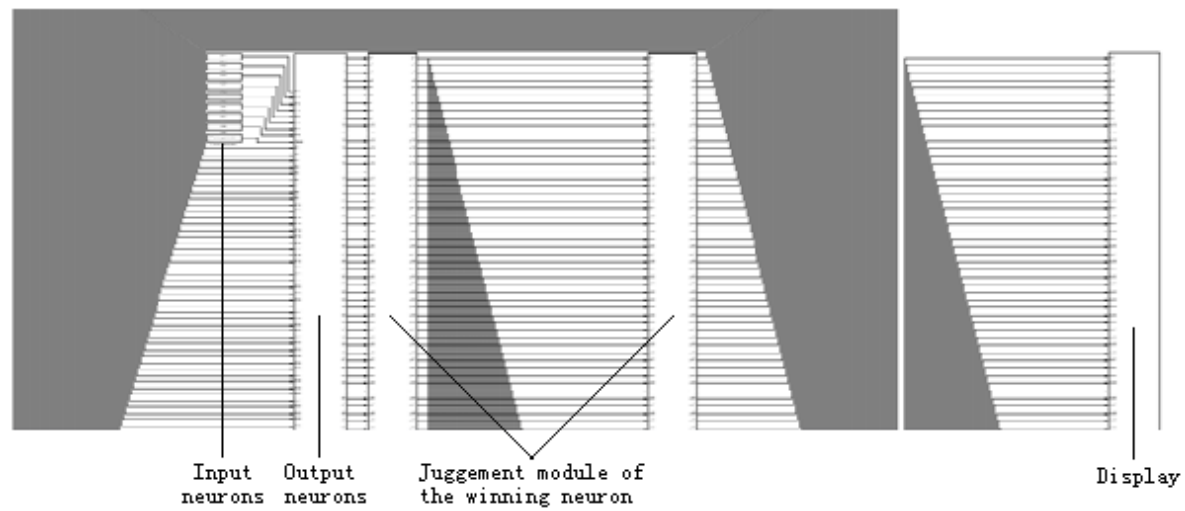

Figure 1. The overall design of memristive neural network

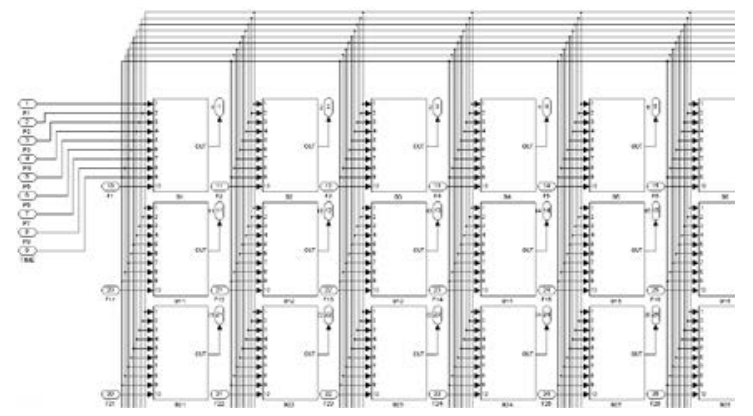

Figure 2. The design of input and output layer

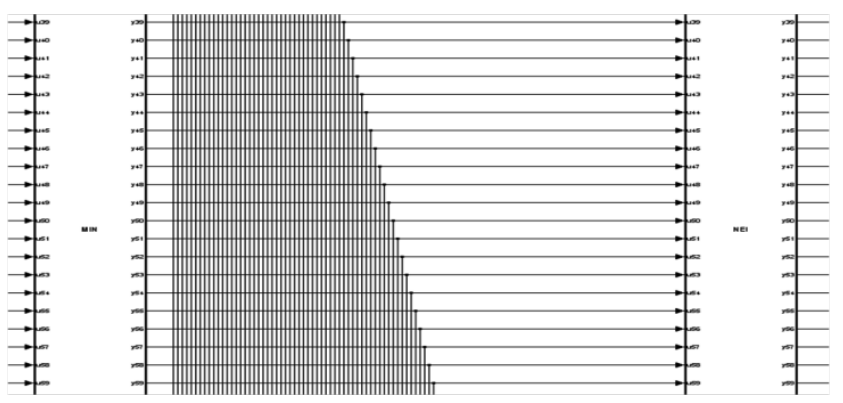

Figure 3. The design of winning neuron judgment module

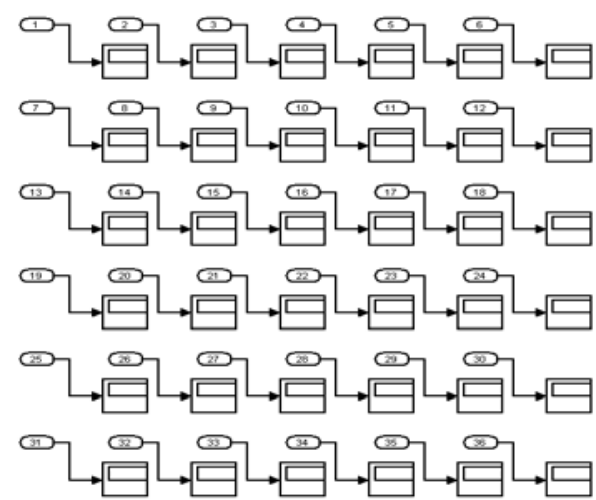

Figure 4. The design of display module

\subsection{Program flow of memristive neural network}

The overall program flow of systems is show in Figure 5. Its main idea is as follows: firstly, test the number of samples and denote as $\mathrm{n}$. Secondly, read a group of fault eigenvectors $\mathrm{P}$ into the input neurons, and find the winning neuron i. Thirdly, Set the timing pulse, if the timing pulse is 1 , determine the neighborhood $\mathrm{Ni}$ by winning neuron $\mathrm{i}$. weights of winner $\mathrm{i}$ and other output neurons in the neighborhood $\mathrm{Ni}$ are trained, and finally display the training result. If the timing pulse is 0 , the system diagnose the fault type only by the trained neural network, no longer modify weights, then the diagnosis result will be displayed to the user. Finally, the number of samples $n$ minus 1 and 
return to the first step, read the next group of fault eigenvectors. Repeat the above steps until all samples processed completely.

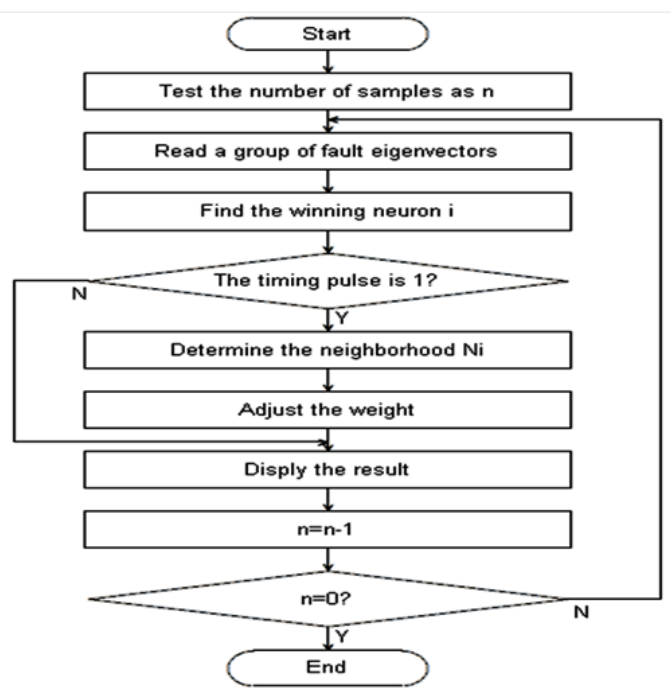

Figure 5. The overall program flow

\section{FAULT DIAGNOSIS OF TRANSFORMER BY MEMRISTIVE NEURAL NETWORK}

This study takes the oil-immersed power transformer as the research object. Dissolved gases analysis is the main method for insulation fault diagnosis of oil-immersed power transformer. The ratio of characteristics gas in transformer oil $\frac{\mathrm{C}_{2} \mathrm{H}_{2}}{\mathrm{C}_{2} \mathrm{H}_{4}}, \frac{\mathrm{CH}_{2}}{\mathrm{H}_{2}}, \frac{\mathrm{C}_{2} \mathrm{H}_{2}}{\mathrm{C}_{2} \mathrm{H}_{8}}$ are entered into memristive neural network as characteristic values P1, P2, P3. The fault types selected include the following: the normal state of operation, low temperature overheat(below 300 degrees Celsius), mid temperature overheat(between 300 and 700 degrees Celsius), high temperature overheat(above 700 degrees Celsius), partial discharge, low energy discharge, low energy discharge and overheat, arc discharge, arc discharge and overheat. Input eigenvectors of standard samples one by one to the neural network, and the common 9 kinds of failures are shown in Table 1.

After repeated training, the memristor will keep changing the value, namely adjusting weights. At the end of training, the memristor will save the last value, and mapping results of the output layer are shown in Figure 6. " 1 ” represents the winning neuron, "0" represents the neuron of failure. After the training, neurons which display " 1 " will be marked by the corresponding code of fault type. Letters from A to I respectively represent the corresponding 9 kinds of fault types. The next step, the test sample is input to the neural network. Compare the position information of the winning neuron and that in Figure 3 and obtain the fault type corresponding to the test sample.

Table 1. Normal sample data

\begin{tabular}{|c|c|c|c|c|}
\hline Fault code & Eigenvector $\mathrm{P}_{1}$ & Eigenvector $\mathrm{P}_{2}$ & Eigenvector $\mathrm{P}_{3}$ & Fault type \\
\hline $\mathrm{A}$ & 0.0700 & 0.2610 & 0.3570 & Normal \\
\hline $\mathrm{B}$ & 0.0000 & 2.6600 & 0.7700 & Low temperature overheat \\
\hline $\mathrm{C}$ & 0.0885 & 1.2000 & 1.8455 & Mid temperature overheat \\
\hline $\mathrm{D}$ & 0.0385 & 1.2550 & 10.2265 & High temperature overheat \\
\hline $\mathrm{E}$ & 0.0220 & 0.0400 & 0.8700 & Partial discharge \\
\hline $\mathrm{F}$ & 3.1200 & 0.4355 & 4.1800 & Low energy discharge \\
\hline $\mathrm{G}$ & 5.2100 & 4.4000 & 5.6900 & $\begin{array}{c}\text { Low energy discharge and } \\
\text { overheat }\end{array}$ \\
\hline $\mathrm{H}$ & 0.4365 & 0.3990 & 12.0055 & Arc discharge \\
\hline $\mathrm{I}$ & 0.1100 & 1.4000 & 15.5900 & Arc discharge and overheat \\
\hline
\end{tabular}




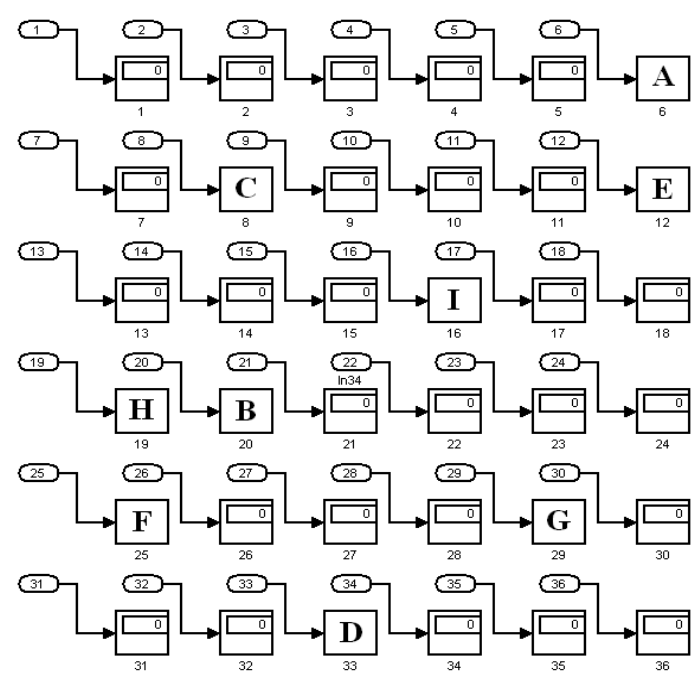

Figure 6. The training result

10 groups of sample data are input to the neural network trained completely one by one. Record the fault type corresponding to each test sample and contrast with the actual fault type. Table 2 shows the fault diagnosis result and actual fault type of the test sample. The diagnosis result shows that No.1, No.2, No.3, No.4, No.5, No.6, No.7, No.8, No.9, No.10 respectively correspond to the fault code I, F, G, H, D, H, D, D, B, C.

From Table 2, fault diagnosis result of the test sample based on memristive neural network fits well with the actual fault type, without errors of judgment. The experimental result shows that this system used to the fault diagnosis of power transformer can get better results.

Table 2. Fault diagnosis result of the test sample

\begin{tabular}{|c|c|c|c|c|c|}
\hline $\begin{array}{c}\text { Serial } \\
\text { number }\end{array}$ & $\begin{array}{c}\text { Eigenvector } \\
\mathrm{P}_{1}\end{array}$ & $\begin{array}{c}\text { Eigenvector } \\
\mathrm{P}_{2}\end{array}$ & $\begin{array}{c}\text { Eigenvector } \\
\mathrm{P}_{3}\end{array}$ & diagnosis result & actual fault \\
\hline 1 & 0.1300 & 1.2600 & 12.8200 & $\mathrm{I}$ & $\mathrm{I}$ \\
\hline 2 & 3.9150 & 0.5500 & 5.1800 & $\mathrm{~F}$ & $\mathrm{~F}$ \\
\hline 3 & 3.1100 & 4.5500 & 6.5900 & $\mathrm{G}$ & $\mathrm{G}$ \\
\hline 4 & 0.2500 & 0.1710 & 1.9450 & $\mathrm{H}$ & $\mathrm{H}$ \\
\hline 5 & 0.0275 & 1.3000 & 9.2200 & $\mathrm{D}$ & $\mathrm{D}$ \\
\hline 6 & 1.1900 & 0.3000 & 6.9320 & $\mathrm{H}$ & $\mathrm{H}$ \\
\hline 7 & 0.0050 & 7.1200 & 8.7715 & $\mathrm{D}$ & $\mathrm{D}$ \\
\hline 8 & 0.0000 & 0.8225 & 17.7930 & $\mathrm{D}$ & $\mathrm{D}$ \\
\hline 9 & 0.0080 & 0.9425 & 2.0090 & $\mathrm{~B}$ & $\mathrm{~B}$ \\
\hline 10 & 0.0075 & 1.0000 & 2.6400 & $\mathrm{C}$ & $\mathrm{C}$ \\
\hline
\end{tabular}

\section{CONCLUSIONS}

This paper presents a new kind of fault diagnosis system that is used to diagnose and analyze the failure information of power transformer. The model of this fault diagnosis system based on memristive neural network is built by MATLAB Simulink, and its weight is achieved through the memristor. Through the comparison between the experimental result and the actual fault type, it shows that this system used to the fault diagnosis of power transformer can get better results.

Memristive neural network not only inherit the advantages of traditional neural network, but also greatly improves the operation speed. In addition, it can save the last weight after power-off. The adaptability of this neural network is stronger for new things, new modes. The way of thinking is closer to the human brain. But the commercial memristor is still in the development of production, so that the hardware circuit cannot be built now. It is a pity, the memristor model in this paper is realized through computer simulation, and can't show its superiority in speed. 


\section{References}

1. Bocheng Bao, Wen Hu, Jianping Xu, Zhong Liu, Ling Zou, 2011. Analysis and Implementation of Memristor Chaotic Circuit. Journal of Physics, 60(12): 120502.

2. Chua L.O, 1971. Memristor-The Missing Circuit Element. IEEE Transactions on Circuit Theory, CT-18(5):507-519.

3. General Administration of Quality Supervision, Inspection and Quarantine of the People's Republic of China (QSIQ), Standardization Administration of the People's Republic of China, 2008. GB/T7252-2001 Transformer Oil Dissolved Gas Analysis and Judgment Guidelines. Beijing: China Standard Press.

4. Guoyong Li, 2005. Intelligent Control and MATLAB Realization. Beijing: Electronic Industry Press.

5. IEC Publication 599, 1978. Interpretation for the Analysis of Gases in Transformers and Other Oil-filled Electrical Equipment in Service.

6. Yueming Wang, Peng Wang, Ying Yang, 2008. Fault diagnosis and repair of transformer. Beijing: Chemical Industry Press. 